\title{
Use of needle-based confocal laser endomicroscopy in the diagnosis of multifocal intraductal papillary mucinous neoplasm with high grade dysplasia
}

A 64-year-old woman with newly diagnosed type 2 diabetes mellitus was admitted with intermittent abdominal pain of 3 years' duration. Computed tomography (CT) showed multifocal pancreatic cystic lesions with a dilated main pancreatic duct and calcification of a cyst wall (ฉ Fig.1), raising suspicion of a mucinous neoplasm of the pancreas.

Needle-based confocal laser endomicroscopy (nCLE) (AQ-Flex 19; Mauna Kea Technologies, France) is a novel imaging technique that enables real time in vivo microscopic imaging of a cyst wall during endoscopy, with a promising diagnostic yield [1-3]. The diagnosis of intraductal papillary mucinous neoplasm (IPMN) is indicated by the presence of finger-like papillae, while mucinous cystic neoplasms (MCNs) have a characteristic single band-like epithelium on nCLE [1 - 3]. We performed endoscopic ultrasoundguided fine-needle aspiration (EUS-FNA) in our patient, with EUS confirming multifocal anechoic cystic lesions extending

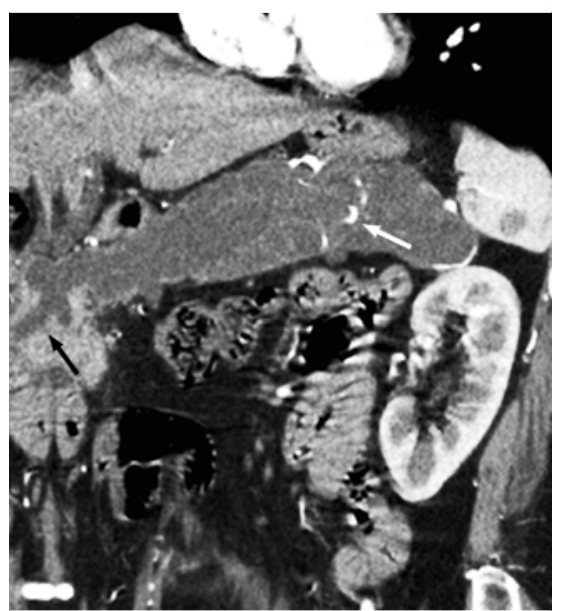

- Fig. 1 Computed tomography (CT) image showing multifocal pancreatic cystic lesions with dilated main pancreatic duct (black arrow) and calcification of cyst wall (white arrow). The patient was a 64-yearold woman with newly diagnosed type 2 diabetes mellitus and with intermittent abdominal pain of 3 years' duration. from the head of the pancreas to the tail, with hyperechoic margins. nCLE showed dark aggregates of cells with surrounding small vessels ( $\triangleright$ Video 1 ), which had indicated features concerning for neoplasia in an earlier study [4]. The cyst fluid had a mucinous appearance and viscosity, and FNA cytology confirmed neoplastic mucinous cells ( $>$ Fig. 2 a, b). The patient consequently underwent a total pancreatectomy ( Fig.3). Histopathological ex- amination revealed an IPMN with high grade dysplasia (\$Fig.4). Lymph node sampling was negative for metastasis.

Wider use of $\mathrm{nCLE}$ has resulted in consensus on some of the characteristic features of common pancreatic cystic lesions, but this is an evolving area with scope for further definition of diagnostic features. In this case, nCLE images showed features concerning for neoplasia prior to surgery. The pattern of dark

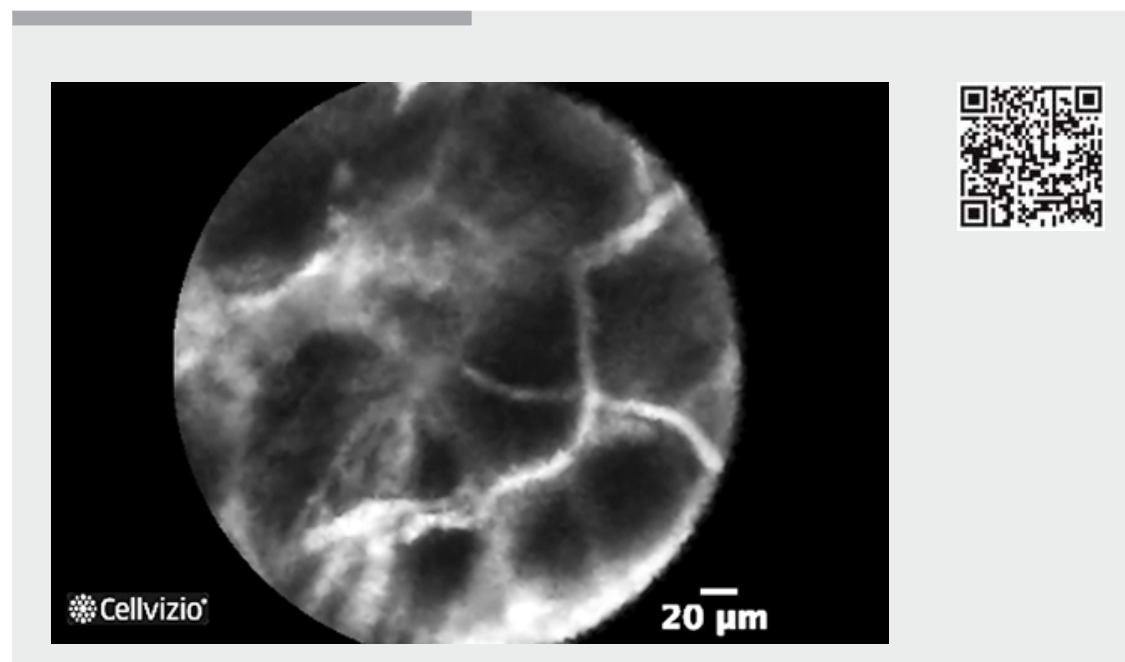

Video 1 Needle-based confocal laser endomicroscopy (nCLE) in the diagnosis of multifocal intraductal papillary mucinous neoplasm with high grade dysplasia: endoscopic ultrasound shows multifocal cystic lesions with hyperechoeic margins, and nCLE shows dark aggregates of cells with surrounding small vessels.
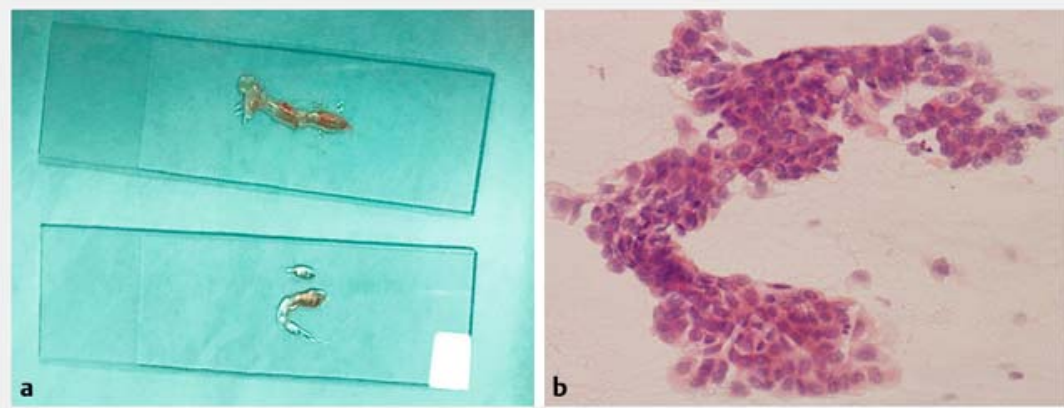

Fig. 2 a Fine needle aspiration (FNA) specimen showing mucinous appearance. b FNA cytology shows neoplastic mucinous cells (hematoxylin and eosin [H\&E], × 200). 


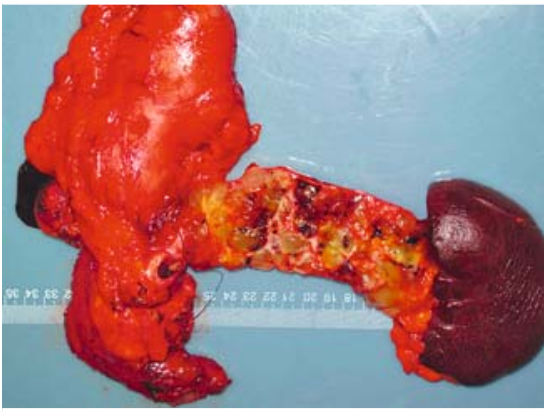

-Fig. 3 The surgically resected specimen. Throughout the pancreas there were multifocal cystic lesions filled with mucus. aggregates of cells surrounded by small vessels may be a promising characteristic in identification of malignant pancreatic cystic lesions (MPCLs). Further studies are required to confirm these findings and to establish nCLE criteria in the diagnosis of MPCLs.

Endoscopy_UCTN_Code_CCL_1AF_2AF_3AC

Competing interests

None

The Authors

Yun-Lu Feng ${ }^{1}$, Ai-Ming Yang ${ }^{1}, X_{i} \mathbf{W u}^{1}$, Zhilan Meng $^{2}$, Xiaoyan Chang ${ }^{2}$, Zhiyong Liang ${ }^{2}$, Junchao Guo $^{3}$

1 Department of Gastroenterology, Peking Union Medical College Hospital, Beijing, China

2 Department of Pathology, Peking Union Medical College Hospital, Beijing, China

3 Department of Surgery, Peking Union Medical College Hospital, Beijing, China

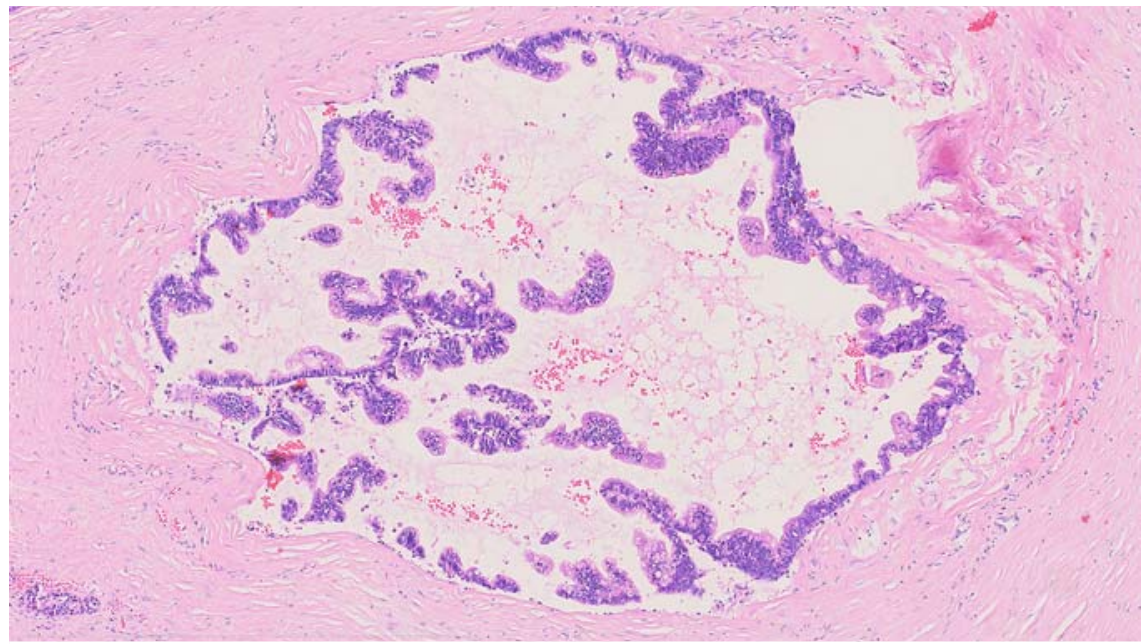

- Fig. 4 Histopathological appearance shows papillae protruding into the cyst lumen and neoplastic mucinous cells arranged in an irregular multilamellar pattern without infiltration (hematoxylin and eosin $[\mathrm{H} \& \mathrm{E}], \times 40)$.

Corresponding author

\section{Ai-Ming Yang, MD}

Department of Gastroenterology,

Peking Union Medical College Hospital,

1 Shuaifuyuan, Wangfujing Avenue, Beijing 100730, China

Fax: +86-10-69155017

yangaiming@medmail.com.cn

\section{Acknowledgment}

The authors thank Sameer Siddique MD, from Einstein Medical Center Philadelphia, United States, for his kind help in language editing.

\section{References}

[1] Nakai Y, Iwashita T, Park DH et al. Diagnosis of pancreatic cysts: EUS guided, throughthe-needle confocal laser-induced endomicroscopy and cystoscopy trial: DETECT study. Gastrointest Endosc 2015; 81: 1204 1214

[2] Napoleon B, Lemaistre Al, Pujol B et al. In vivo characterization of pancreatic cystic lesions by needle-based confocal laser endomicroscopy (nCLE): proposition of a comprehensive $\mathrm{nCLE}$ classification confirmed by an external retrospective evaluation. Surg Endosc 2016; 30: 2603-2612

[3] Napoleon B, Lemaistre AI, Pujol B et al. A novel approach to the diagnosis of pancreatic serous cystadenoma: needle-based confocal laser endomicroscopy. Endoscopy 2015; 47: 26-32

[4] Konda V], Meining A, Jamil LH et al. A pilot study of in vivo identification of pancreatic cystic neoplasms with needle-based confocal laser endomicroscopy under endosonographic guidance. Endoscopy 2013; 45 : $1006-1013$

\section{Bibliography}

DOI https://doi.org/10.1055/s-0043-115889

Published online: 3.8.2017

Endoscopy 2017; 49: E277-E278

(c) Georg Thieme Verlag KG

Stuttgart · New York

ISSN 0013-726X

\section{ENDOSCOPY E-VIDEOS \\ https://eref.thieme.de/e-videos}

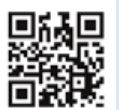

Endoscopy E-Videos is a free access online section, reporting on interesting cases and new techniques in gastroenterological endoscopy. All papers include a high quality video and all contributions are freely accessible online.

This section has its own submission website at

https://mc.manuscriptcentral.com/e-videos 